\title{
Endosialin-expressing bone sarcoma stem-like cells are highly tumor-initiating and invasive
}

\author{
DONG-XIU SUN ${ }^{1 *}$, GUANG-JUN LIAO ${ }^{2 *}$, KE-GUI LIU ${ }^{3}$ and HAN JIAN ${ }^{2}$ \\ Departments of ${ }^{1}$ Operating Room and ${ }^{2}$ Orthopaedic Surgery; ${ }^{3}$ The Joint Department of Orthopaedics, \\ Yantaishan Hospital, Yantai, Shandong 264000, P.R. China
}

Received October 12, 2014; Accepted June 15, 2015

DOI: $10.3892 / \mathrm{mmr} .2015 .4218$

\begin{abstract}
It has been reported that the presence of a small group of cancer stem-like 'side population (SP)' cells is responsible for therapy failure and tumor recurrence. The present study demonstrated that primary human osteosarcoma samples contained a SP of about $3.9 \%$ which overexpressed $\mathrm{ABC}$ transporters, including $\mathrm{ABCA} 1, \mathrm{ABCB} 1, \mathrm{ABCB} 2$ and ABCG2, which are associated with drug resistance and may have contributed to multi-drug resistance of SP cells. Furthermore, these SP cells displayed increased expression of endosialin (CD248) and other stem cell surface proteins, including CD133, octamer-binding transcription factor 3/4A, Nanog and Nestin, which are ultimately responsible for high self-renewal and deregulated cell proliferation. In addition, it was shown that endosialin-overexpressing SP cells were able to regenerate the tumor population and had a high invasive potential. Therefore, the present study suggested that osteosarcoma SP cells were cancer stem cells, as they displayed stem-like properties; furthermore, endosialin may be a potential target to prevent osteosarcoma recurrence following chemotherapy.
\end{abstract}

\section{Introduction}

Tumors are heterogeneous and contain sub-sets of cancer cells which may be resistant to cancer treatment and possess tumor-initiating and re-populating abilities; they promote cancer progression, are invasive, and are termed as cancer stem cells (CSCs). These CSCs have long-term survival capacity and are responsible for tumor recurrence, invasiveness and metastasis (1). Therefore, molecular characterization of CSCs and the elucidation of their targeted downstream signaling

Correspondence to: Professor Ke-Gui Liu, The Joint Department of Orthopaedics, Yantaishan Hospital, 91 Jiefang Road, Yantai, Shandong 264000, P.R. China

E-mail: liukegui324@sina.com

*Contributed equally

Key words: endosialin, side population, drug resistance, self-renewal, adenosine triphosphate binding cassette transporters pathways are essential for designing novel anti-cancer drugs. A number of studies showed that the Hoechst 33342 dye exclusion assay is a valuable method to identify and to study the characteristics of CSCs. The small population of cancer cells which excludes the Hoechst 33342 dye are termed as the side population (SP) cells according to their separate appearance in fluorescence-associated cell sorting (FACS) dot plots. SP cells were first identified among bone marrow cells and in recent decades, SP cells were isolated and characterized in several types of cancer (2-4). These SP cells were either reduced or disappeared completely upon treatment with verapamil, which confirmed that resistance-associated drug efflux is mediated by the overexpression of cell surface multidrug resistance protein 1/P-glycoprotein and/or breast cancer resistance protein 1/adenosine triphosphate binding cassette (ABC)G2, which contributed to chemoresistance and tumor relapse $(2,5)$. Of note, these SP cells have been shown have elevated levels of stem cell surface proteins, including CD133, CD44, octamer-binding transcription factor (Oct)-4 and Nanog, which have a major role in tumor re-population and their invasion $(3,4)$. In addition, increased expression of novel cell surface protein endosialin was found to be frequently associated with mesenchymal tumors (6). Furthermore, it was shown that SP cells were able to initiate tumors in NOD/SCID mice, even at low doses (7).

Bone sarcomas are generally rare types of tumor and are usually derived from either mesenchymal tissues or non-epithelial tissues. Osteosarcoma, one of the most common bone sarcoma types, occurs as a high-grade tumor and has a tendency to develop pulmonary metastases (8). The mean survival time of osteosarcoma patients after diagnosis of pulmonary metastases is less than five years (9). Despite recent advances in cancer treatment strategies, there has not been any significant improvement in the survival rate of osteosarcoma patients. It was reported that presence of cancer stem-like SP cells in bone sarcoma is responsible for therapy resistance, tumor recurrence, invasion and metastasis $(10,11)$. However, the underlying molecular mechanism of the drug resistance and tumor relapse has not been fully elucidated. SP cells have been detected in various types of cancer, including leukemia and medulloblastoma as well as colorectal, hepatocellular, prostate, thyroid, breast and ovarian carcinoma $(7,12)$. Only a small number of studies detailing the molecular characterization of sarcoma SP cells are currently available $(13,14)$. Consequently, 
the present study was performed to isolate and characterize cancer stem-like SP cells from osteosarcoma samples. SP cells were analyzed for their expression of endosialin and other stem cell surface proteins associated with drug resistance, as well as for their clonogenicity and invasiveness, which is essential for re-population and formation of metastasis.

\section{Materials and methods}

Tumor samples and cell culture. Human osteosarcoma samples were obtained from the patients at the time of surgery in the Department of Orthopaedic Surgery, Yantaishan Hospital (Yantai, China). Tumor samples were prepared according to the ethical procedures approved by Yantaishan Hospital, which approved the study. 3. Written informed consent was obtained from the patient. Patient details: $n=10$; tumor grade, high; region, osteoblastic osteosarcoma; stage, III with lung metastasis; age, 30-39; no previous chemotherapy. After isolation, the samples were extensively washed in phosphate-buffered saline (PBS; Sigma-Aldrich, St. Louis, MO, USA) containing antibiotics and incubated overnight in Dulbecco's modified Eagle's medium (DMEM)/F12 (Gibco-BRL, Invitrogen Life Technologies, Carlsbad, CA, USA) containing penicillin $(500 \mathrm{U} / \mathrm{ml})$, streptomycin $(500 \mu \mathrm{g} / \mathrm{ml})$ and amphotericin B $(1.25 \mu \mathrm{g} / \mathrm{ml}$; Gibco-BRL). Enzymatic digestion was performed using collagenase (1.5 mg/ml; Gibco-BRL) and hyaluronidase (20 $\mu \mathrm{g} / \mathrm{ml}$; Gibco-BRL) in PBS for $1 \mathrm{~h}$. Cells were cultured in DMEM with $10 \%$ FBS, supplemented with antibiotics and maintained in $\mathrm{T}-75$ flasks at $37^{\circ} \mathrm{C}$ in a humidified atmosphere of $5 \% \mathrm{CO}_{2}$ and $95 \%$ air. On becoming $90 \%$ confluent, cells were removed from culture flasks using Trypsin-EDTA (0.25\%-53 mM EDTA; Sigma-Aldrich), washed and suspended in $10 \%$ DMEM. Cells were counted using a hemocytometer.

FACS analysis. Control group: Cells + Hoechst 33342 dye $(n=9)$; Drug-treatment group: cells + Verapamil + Hoechst 33342 dye $(n=9)$. Approximately $10^{6}$ cells $/ \mathrm{ml}$ in DMEM with 10\% FBS were labeled with Hoechst 33342 stock (Sigma-Aldrich) with bis-benzimide ( $5 \mu \mathrm{l} / \mathrm{ml}$; Sigma-Aldrich) either with dye alone or in combination with drug (Verapamil; $0.8 \mu \mathrm{l} / \mathrm{ml}$; Sigma-Aldrich). Cells were re-suspended in $500 \mu \mathrm{l} \mathrm{Hank's} \mathrm{balanced} \mathrm{salt} \mathrm{solution} \mathrm{containing} 10 \mathrm{mM}$ 4-(2-hydroxyethyl)-1-piperazineethanesulfonic acid (Sigma-Aldrich) for FACS analysis. Finally, cells were counterstained with propidium iodide (PI; $2 \mu \mathrm{g} / \mathrm{ml}$; Sigma-Aldrich).

Sphere formation assay. The SP and non-SP cells were seeded at a density of $\sim 60,000$ cells/well in six-well, ultra low attachment plates under serum-free, sphere-specific conditions as described previously (15). After six days of culturing, the sarcospheres were counted and analyzed for the expression of stemness proteins by fluorescence microscopy (EVOS FL Cell Imaging system; Life Technologies).

Immunofluorescent staining. The sorted SP and non-SP cells of OS-55 were fixed in BD Cytofix solution (BD Biosciences, Franklin Lakes, NJ, USA) and incubated for $20 \mathrm{~min}$ at $4^{\circ} \mathrm{C}$. After 20 min blocking in donkey serum, cells were incubated with goat anti-Oct3/4A antibody (sc-8628; Santa Cruz Biotechnology, Inc., Dallas, TX, USA) overnight at $4^{\circ} \mathrm{C}$, and subsequently incubated with either Rhodamine red or fluorescein isothiocyanate (FITC)-conjugated donkey anti-goat antibody (1:200; Jackson Immunoresearch Laboratories, West Grove, PA, USA). For CD133 and Nanog immunofluorescence analysis, cells were stained with FITC-conjugated anti-human Nanog (cat. no. sc-33760; 1:500; Santa Cruz Biotechnology, Inc.) or CD44 antibody (1:5; eBioscience, San Diego, CA, USA). Human embryonic stem cells were used as a positive control (Invitrogen Life Technologies). Similarly, the sarcospheres were stained with rabbit monoclonal CD133 (cat. no. 141203; 1:500; BioLegend, Inc., San Diego, CA, USA) and rabbit monoclonal Oct-3/4A (cat. no. A13998; 1:500; Life Technologies) for $90 \mathrm{~min}$. For endosialin staining, 100,000 cells were seeded on a coverslip and after $24 \mathrm{~h}$ of incubation, cells were stained for endosialin with rabbit anti-human endosialin polyclonal primary antibody (cat. no. HPA051856; 1:500; Sigma-Aldrich) for $90 \mathrm{~min}$. After washing with PBS, cells were incubated with FITC-conjugated secondary antibody. The cells were observed under a confocal microscope (Leica TCS SP8 STED 3X; Leica, Wetzlar, Germany) and all images were analyzed and processed using Adobe Photoshop CS6 (Microsoft Corp., Redmont, WA, USA).

Drug sensitivity assay. Approximately $1 \times 10^{3}$ cells/plate were cultured in 96-well plates and treated with the chemotherapeutic drugs $1 \mu \mathrm{M}$ doxorubicin, $10 \mu \mathrm{M}$ cisplatin or $1 \mu \mathrm{M}$ methotrexate (Sigma-Aldrich) for $48 \mathrm{~h}$ as previously described (15). The mean optical density value at $450 \mathrm{~nm}\left(\mathrm{OD}_{450}\right)$ obtained was represented as a graph. The drug resistance in the groups was calculated using the following formula: Cell resistance rate $(\%)=\left(\right.$ experimental group $\mathrm{OD}_{450} /$ control group $\left.\mathrm{OD}_{450}\right) \times 100$. The values presented in the graph are the average of three independent experiments.

Reverse transcription quantitative polymerase chain reaction $(R T-q P C R)$ analysis. The RNA was isolated using an RNA isolation kit (PureLink RNA mini kit; Invitrogen Life Technologies) and the RNA samples were treated with DNase I to exclude DNA contamination. The following PCR primers $(15,16)$ were used for RT-qPCR analysis: CD133 forward, 5'-TCTTGACCGACTGAGAC-3' and reverse, 5'-ACT TGATGGATGCACCAAGCAC-3'; Nestin forward, 5'-GAC GGAGGAGGTAGCCCGCA-3' and reverse, 5'-GCCTCCACA GCCAGCTGGAACT-3'; ABCG2 forward, 5'-TCAATCAAA GTGCTTCTTTTTTATG-3' and reverse, 5'-TTGTGGAAG AATCACGTGGC-3'; Nanog forward, 5'-TCCTCCTCTTCC TCTATACTAAC-3' and reverse, 5'-CCCACAAATCACAGG CATAG-3'; GAPDH forward, 5'-TGGACTCCACGACGTACT CAG-3' and reverse, 5'-ACATGTTCCAATATGATTCCA-3'; OCT3/4 forward, 5'-ACATGTGTAAGCTGCGGCC-3' and reverse, 5'-GTTGTGCATAGTCGCTGCTTG-3'. GAPDH was used as an internal control. All primers were synthesized by Shanghai Generay Biotech Co., Ltd. (Shanghai, China). PCR cycling conditions were as follows: $95^{\circ} \mathrm{C}$ for $2 \mathrm{~min}, 40$ cycles of $95^{\circ} \mathrm{C}$ for $30 \mathrm{sec}, 55-60^{\circ} \mathrm{C}$ for $1 \mathrm{~min}$ and $72^{\circ}$ for $30-60 \mathrm{sec}$ using the Bio-Rad T100 thermal cycler (Bio-Rad Laboratories, Inc., Hercules, CA, USA). PCR products were electrophoresed on a $1.2 \%$ agarose gel and stained with ethidium bromide (Sigma-Aldrich). The band intensities were quantified using ImageJ software version 1.0 (National Institutes of Health, 
Bethesda, MD, USA). The values presented in the graph are the average values of three independent experiments.

Western blot analysis. Proteins were extracted from the SP and non-SP cells, and the protein concentration was determined using the Bradford assay. Following 12\% SDS-PAGE (Bio-Rad Laboratories, Inc.) and transfer to a polyvinylidene difluoride membrane (Bio-Rad Laboratories, Inc.), the gels were treated with the primary antibodies: Rabbit anti-human polyclonal $\beta$-catenin (cat. no. sc-7199; 1:500; Santa Cruz Biotechnology, Inc.), rabbit polyclonal ABCG2 (cat. no. AV43649; 1:500; Sigma-Aldrich) and rabbit polyclonal GAPDH (cat. no. sc-25778; 1:500; Santa Cruz Biotechnology, Inc.) for $90 \mathrm{~min}$, the secondary antibody (goat anti-rabbit immunoglobulin $\mathrm{G}$ with alkaline phosphatase markers) and a chemiluminescence reagent (Life Technologies, Carlsbad, CA, USA). Blots were detected and scanned using a densitometer (GS-710; Bio-Rad Laboratories, Inc.).

Invasion assay. Cellular invasiveness of SP and non-SP cells was determined using six-well Matrigel invasion chambers (BD Biosciences). Cells were seeded in serum-free medium at a density of $2 \times 10^{5}$ per insert. Outer wells were filled with DMEM containing 5\% FBS as chemoattractant and incubated at $37^{\circ} \mathrm{C}$ for $48 \mathrm{~h}$. Subsequently, the non-invaded cells were removed from the top layer of the Matrigel with a cotton bud (17). The membrane containing the invaded cells was stained with hematoxylin for $3 \mathrm{~min}$ (Sigma-Aldrich), washed and mounted on slides. The entire membrane with invading cells was counted under a light microscope at 40x objective. The values represented in the graph are the average value of two independent experiments.

Statistical analysis. All statistical analyses were performed with the SPSS 20.0 package (IBM SPSS, Armonk, NY, USA). Data are presented as the mean $\underline{ \pm}$ standard deviation. Statistical differences between the patient and control groups were assessed using a t-test and an analysis of variance. $\mathrm{P}<0.01$ was considered to indicate statistically significant differences.

\section{Results}

SP cells are present in human osteosarcoma samples. Human osteosarcoma samples obtained from the patients were analyzed for the presence of SP cells by the FACS-based Hoechst 33342 dye exclusion method. In the FACS dot plot, a 3.9\%-population of SP cells was gated in the lower left quadrant (Fig. 1A). After treatment with Verapamil, an inhibitor of ABC transporters, the population of SP cells was diminished to $0.7 \%$ (Fig. 1B and $\mathrm{C}$ ). Therefore, these results confirmed the role of the $\mathrm{ABC}$ transporter ABCG2 in treatment resistance-associated drug expulsion by SP cells.

Endosialin (CD248) and other stemness proteins are elevated in osteosarcoma SP cells. It was previously shown that in osteosarcoma cell lines, endosialin (CD248) was expressed in SP cells (18). Hence, in the present study, the osteosarcoma SP cells identified by FACS were validated for the expression of endosialin and other stem-cell surface proteins. As shown in Fig. 2A, endosialin expression was elevated in SP cells
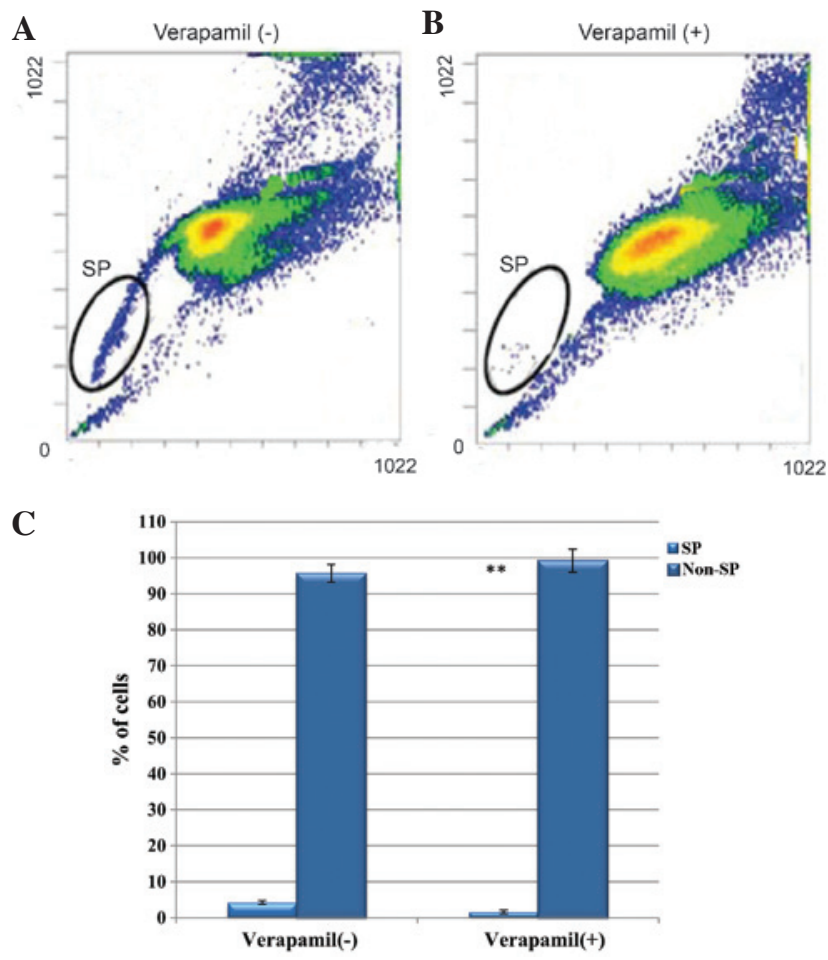

Figure 1. Identification of SP cells from human osteosarcoma. Live cells were selected against propidium iodide staining. (A) Dot plot of FACS analysis showing the presence of $3.9 \%$ of SP cells (gated population). (B) After treatment with verapamil (adenosine triphosphate binding cassette transporter inhibitor) treatment, the SP cell population was diminished to $0.7 \%$. (C) Results of FACS analysis quantified from three independent experiments. ${ }^{* *} \mathrm{P}<0.01$ for non-SP vs. SP. SP, side population; FACS, fluorescence-associated cell sorting.

compared with that in non-SP cells. Similarly, stem cell surface proteins, including CD133, Oct-3/4A (one of the isoforms of Oct-4 proteins) and Nanog were markedly elevated in SP cells (Fig. 2A). The increased expression of the abovementioned stem cell proteins in SP cells were thought to be involved in the maintenance of self-renewal and the high proliferation rate of SP cells. Next, the present study investigated the transcriptional upregulation of these genes by RT-PCR. The relative mRNA expression of endosialin, Nestin, Oct-3/4A and Naong was significantly elevated in SP cells compared with that in non-SP cells (Fig. 2B). In addition, immunofluorescence microscopy revealed that the osteosarcoma SP cells showed positivity for CD133, Oct3/4A (punctate nuclear staining), endosialin and Nanog (punctate nuclear staining), whereas the non-SP cells showed either low or null expression (Fig. 3).

Osteosarcoma SP cells have self-renewal capacity. One of the most important characteristic features of SP cells is their ability to self-renew. It was reported that side population cells from several solid tumors have a high proliferation rate and are able to efficiently propagate the tumor population. In order to assess the self-renewal capacity of SP cells, a clonogenic formation efficiency test was performed. After 6 days of culturing, it was observed that the purified SP cells were able to generate significantly more clusters of tumor spheres (sarcospheres) than non-SP cells (Fig. 4A). Furthermore, the sarcospheres generated by SP cells were bigger in size when compared to the non-SP spheres (Fig. 4B). Of note, these sarcospheres 

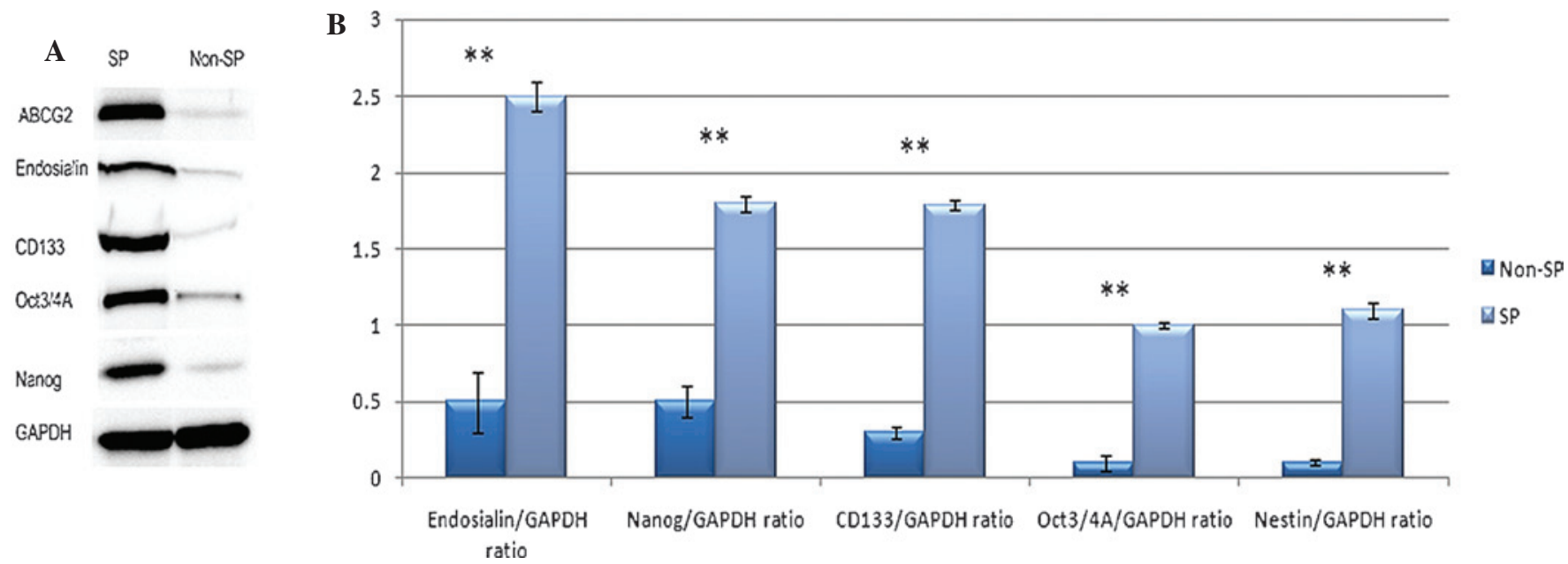

Figure 2. Comparison of gene and protein expression levels between SP and non-SP cells. (A) Western blots showing elevated expression of endosialin and stem cell surface proteins in SP cells. (B) Quantified results of reverse-transcription quantitative polymerase chain reaction analysis for expression of endosialin and other stemness genes in SP and non-SP cells. The elevated mRNA expression of endosialin, CD133, nestin, Oct-3/4A and Nanog are significantly higher in SP cells. The bar represents the standard deviation and ${ }^{* *} \mathrm{P}<0.01$ for non-SP vs. SP. SP, side population; ABC, adenosine triphosphate binding cassette; Oct, octamer-binding transcription factor.

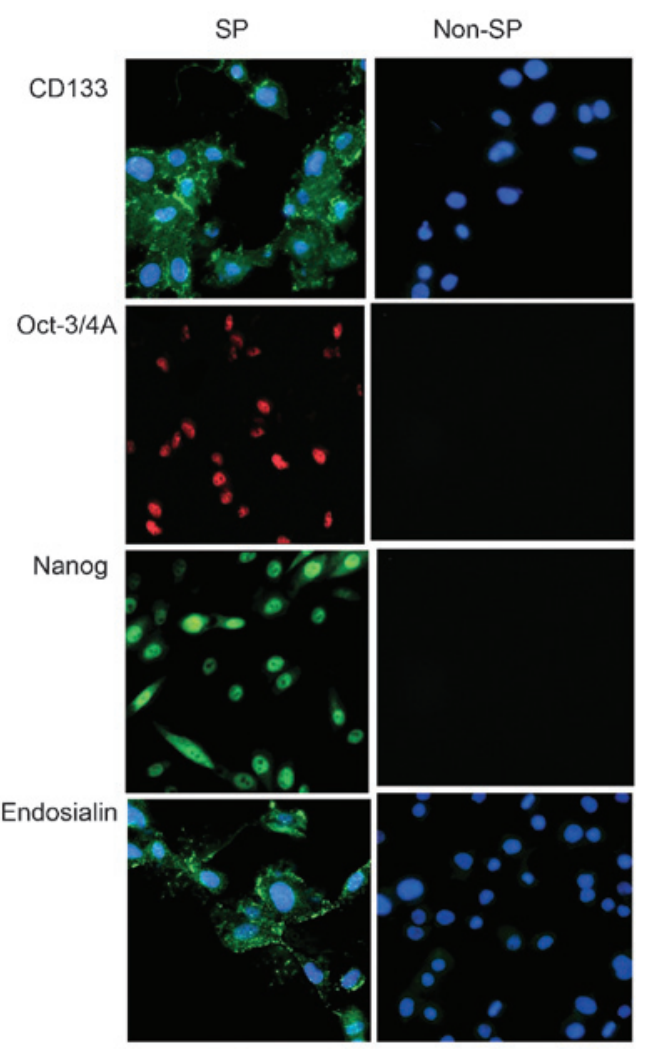

Figure 3. Representative immunofluorescent staining images of sorted SP cells showing immunofluorescence positive to endosialin, CD133, Nanog and Oct3/4A (magnification, x100). Nuclei were stained with DAPI. SP, side population; Oct, octamer-binding transcription factor.

were positive for stem cell surface markers, including CD133 and Oct-3/4A (Fig. 3C). Hence, these results clearly suggested that elevated expression of stem cell proteins in SP cells has a major role in tumor initiation and re-population.

Osteosarcoma SP cells show increased drug resistance and high invasiveness. SP cells were shown to resist several drugs
A
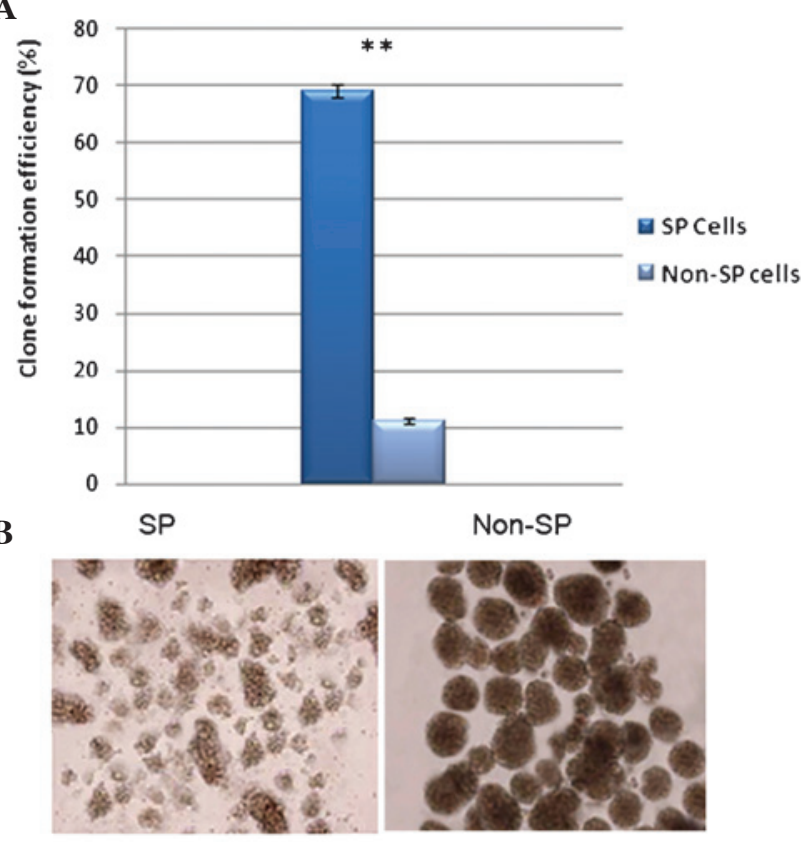

C

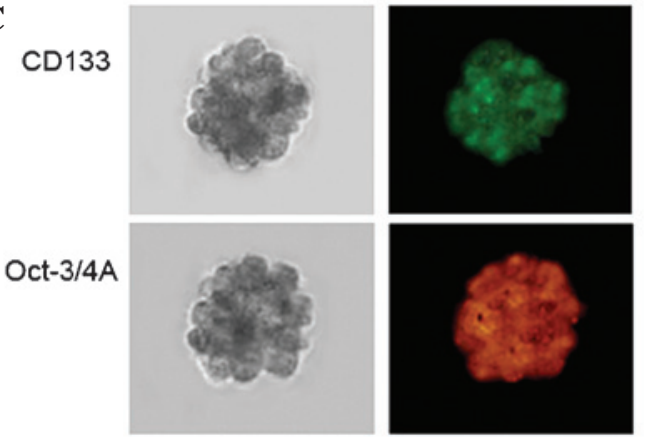

Figure 4. Determination of clone formation efficiency of osteosarcoma SP cells. (A) The SP cells generate sarcospheres rapidly and significantly higher than non-SP cells. Values are expressed as the mean \pm standard deviation. ${ }^{* *} \mathrm{P}<0.01$ for non-SP vs. SP. (B) Representative phase contrast images showing that SP cell-derived sarcospheres are bigger in size than those of non-SP cells. (C) Fluorescence microscopic images showing that SP cell-derived sarcospheres are CD133- and Oct3/4A-positive (magnification, x100). SP, side population; Oct, octamer-binding transcription factor. 


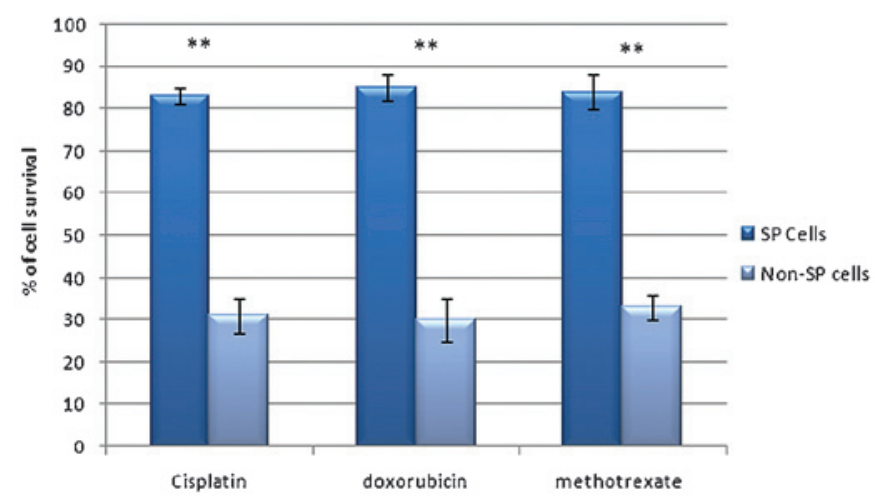

Figure 5. Drug sensitivity assay. SP cells showed an increased drug resistance and an enhanced survival rate after treatment with doxorubicin, cisplatin or methotrexate. Values are expressed as the mean \pm standard deviation of three independent experiments. ${ }^{* *} \mathrm{P}<0.01$ for non-SP vs. SP. SP, side population.

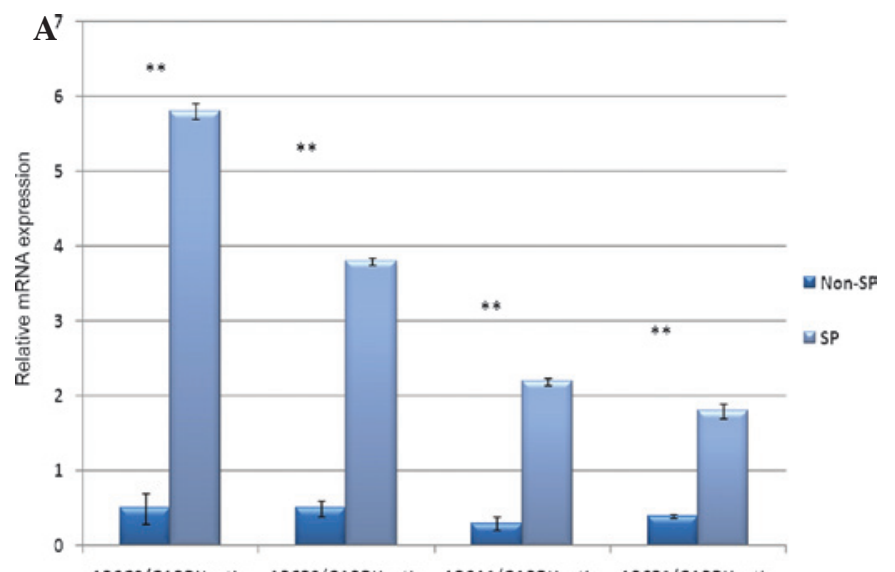

B

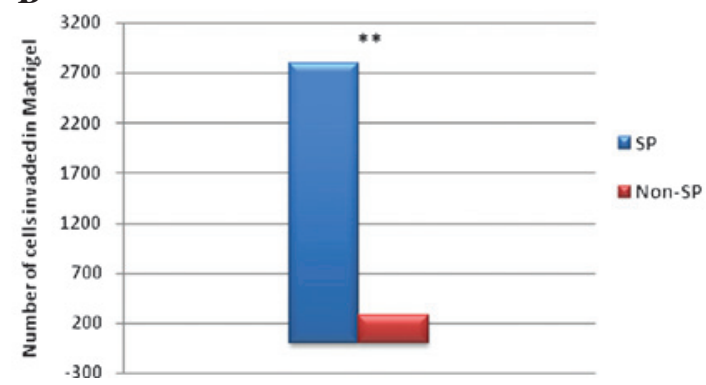

Figure 6. Relative mRNA expression of genes in SP and non-SP cells. (A) Quantified results of the reverse transcription quantitative polymerase chain reaction analysis of $\mathrm{ABC}$ transporter genes. $\mathrm{ABCA} 2, \mathrm{ABCB} 1, \mathrm{ABCC} 1$ and ABCG2 were significantly upregulated in SP cells. (B) SP-cell invasiveness as determined by a Matrigel assay. Graph showing the number of cells invaded across the membrane. ${ }^{* *} \mathrm{P}<0.01$ for non-SP vs. SP. SP, side population; $\mathrm{ABC}$, adenosine triphosphate binding cassette.

and thus, to be responsible for treatment failure and tumor relapse. In order to test the drug resistance capacities of SP and non-SP cells, a drug resistance assay was performed. After treatment with the drugs doxorubicin, cisplatin and methotrexate, the survival rate of SP cells was $>80 \%$, whereas it was $<30 \%$ for non-SP cells (Fig. 5A). These results confirmed that SP cells are highly resistant to several chemotherapeutic drugs. Next, the present study investigated the cause for chemoresistance of SP cells. From Fig. 1 it is clear that dye exclusion of
SP cells actively involves the upregulation of $A B C$ transporter proteins. Therefore, the present study performed a comprehensive RT-qPCR analysis of SP and non-SP cells to evaluate the transcriptional upregulation of various $\mathrm{ABC}$ transporters including $\mathrm{ABCA} 1, \mathrm{ABCB} 1, \mathrm{ABCA} 2$ and $\mathrm{ABCG} 2$. As shown in the graph in Fig. 6A, all of the assessed $\mathrm{ABC}$ transporter genes were markedly upregulated in SP cells, indicating that they are responsible for the drug resistance of osteosarcoma by actively pumping chemotherapeutic drugs out of the SP cells. Furthermore, in order to determine the invasion potential of SP cells, a Matrigel invasion assay was performed. The results showed that SP cells have an enhanced invasive capacity compared with that of non-SP cells (Fig. 6B). Hence, these findings suggested that osteosarcoma SP cells display marked drug resistance and have tumor-initiating as well as invasive properties.

\section{Discussion}

SP cells represent cancer stem cells, as they are able to initiate tumors and hence, they are termed as 'enriched cancer stem cells' (3). The detection of cancer stem-like SP cells in various cancer types as well as in cancer cell lines confirms their role in treatment failure and recurrence of tumors $(19,20)$. Furthermore, several studies reported that SP and non-SP cells have different growth rates and that SP cells are highly tumorigenic in vivo as well as in vitro $(4,21)$. Therefore, the elucidation of crucial pathways mediated by SP cells which are accountable for tumor relapse is essential for designing novel and effective therapeutics. In the present study, primary human osteosarcoma samples from patients were assessed for the presence of SP cells and stem-cell surface markers. Using a Hoechst dye exclusion technique, the presence of an SP population of 3.9\% was detected, which was enriched in stem-cell surface markers as well as endosialin. In line with the findings of the present study, previous studies on osteosarcoma cell lines reported that presence of SP cells with stem-like properties $(15,16)$. Furthermore, the present study showed that gene transcriptional levels of endosialin, CD133, nanog, nestin and Oct-3/4A were markedly upregulated in SP cells. The SP cells were also shown to be positive for these markers by immunofluorescence microscopy. These results clearly suggested that the upregulation of these proteins is, at least in part, responsible for the high proliferation and maintenance of self-renewal of cancer stem-like SP cells in osteosarcoma. This was further confirmed by a sphere formation assay, in which the SP cells were able to generate an increased number of sarcospheres and they were big in size when compared to spheres derived from non-SP cells. Similar to previous studies $(22,23)$, the present study showed that SP cell-derived sarcospheres displayed high expression levels of CD133 and Oct-3/4A. Furthermore, SP cells showed high resistance to several therapeutic drugs as well as an enhanced survival rate. The multi-drug resistance properties of SP cells actively involves the overexpression of several $\mathrm{ABC}$ transporter proteins. The relative mRNA expression levels of $\mathrm{ABC}$ transporter genes were markedly elevated in SP cells compared with those in non-SP cells; ABC transporters are thus accountable for the resistance of osteosarcoma SP cells to DNA-damaging and other anti-cancer agents by efficiently pumping them out of the cells. Another mechanism 
of resistance may be the reduced rate of apoptosis of SP cells, leading to an enhanced survival rate of SP cells after drug treatment. All of these results suggested that osteosarcoma SP cells from patient samples possessed a high capacity of multidrug resistance, clonogenicity and self-renewal in vitro.

In conclusion, the present study clearly indicated that identification and characterization of SP cells is crucial for the elucidation of the underlying molecular mechanisms of SP cell-mediated tumorigenesis. In addition to other studies, the present study showed that human osteosarcoma SP cells expressed elevated levels of endosialin, which suggested that endosialin may be utilized as a potential marker for the purification of SP cells. Since endosialin-expressing SP cells are highly drug resistant and posses self-renewing and invasive properties, it was speculated that endosialin may also be a potential target for designing novel anti-cancer drugs, which aim to eradicate cancer stem cells. However, the precise mechanism and function of elevated endosialin in SP cells requires to be elucidated in further studies.

\section{References}

1. Nguyen DX, Bos PD and Massagué J: Metastasis: From dissemination to organ-specific colonization. Nat Rev Cancer 9: 274-284, 2009.

2. Goodell MA, Brose K, Paradis G, Conner AS and Mulligan RC: Isolation and functional properties of murine hematopoietic stem cells that are replicating in vivo. J Exp Med 183: 1797-1806, 1996.

3. Moserle L, Ghisi M, Amadori A and Indraccolo S: Side population and cancer stem cells: Therapeutic implications. Cancer Lett 288 $1-9,2010$.

4. Wu C and Alman BA: Side population cells in human cancers. Cancer Lett 268: 1-9, 2008.

5. Liu FS: Mechanisms of chemotherapeutic drug resistance in cancer therapy-a quick review. Taiwan J Obstet Gynecol 48: 239-244, 2009.

6. Rouleau C, Curiel M, Weber W, Smale R, Kurtzberg L, Mascarello J, Berger C, Wallar G, Bagley R, Honma N, et al: Endosialin protein expression and therapeutic target potential in human solid tumors: Sarcoma versus carcinoma. Clin Cancer Res 14: 7223-7236, 2008

7. Wu C, Wei Q, Utomo V, Nadesan P, Whetstone H, Kandel R, Wunder JS and Alman BA: Side population cells isolated from mesenchymal neoplasms have tumor initiating potential. Cancer Res 67: 8216-8222, 2007.
8. Helman LJ and Meltzer P: Mechanisms of sarcoma development. Nat Rev Cancer 3: 685-694, 2003.

9. Akiyama T, Dass CR and Choong PF: Novel therapeutic strategy for osteosarcoma targeting osteoclast differentiation, bone-resorbing activity and apoptosis pathway. Mol Cancer Ther 7: 3461-3469, 2008

10. Helman LJ and Meltzer P: Mechanisms of sarcoma development. Nat Rev Cancer 3: 685-694, 2003.

11. Tang N, Song WX, Luo J, Haydon RC and He TC: Osteosarcoma development and stem cell differentiation. Clin Orthop Relat Res 466: 2114-2130, 2008.

12. Dean M, Fojo T and Batess: Tumour stem cells and drug resistance. Nat Rev Cancer 5: 275-784, 2005.

13. Ponti D, Costa A, Zaffaroni N, Pratesi G, Petrangolini G, Coradini D, Pilottis, Pierotti MA and Daidone MG: Isolation and in vitro propagation of tumorigenic breast cancer cells with stem/progenitor cell properties. Cancer Res 65: 5506-5511, 2005.

14. Fang D, Nguyen TK, Leishear K, Finko R, Kulp AN, Hotzs S, Van Belle PA, Xu X, Elder DE and Herlyn M: A tumorigenic subpopulation with stem cell properties in melanomas. Cancer Res 65: 9328-9337, 2005.

15. Yang M, Yan M, Zhang R, Li J and Luo Z: Side population cells isolated from human osteosarcoma are enriched with tumor-initiating cells. Cancer Sci 102: 1774-1781, 2011.

16. Wang L, Park P and Lin CY: Characterization of stem cell attributes in human osteosarcoma cell lines. Cancer Biol Ther 8: 543-552, 2009.

17. Ho MM, Ng AV, Lams and Hung JY: Side population in human lung cancer cell lines and tumors is enriched with stem-like cancer cells. Cancer Res 67: 4827-4833, 2007.

18. Rouleau C, Sancho J, Campos-Rivera J and Teicher BA: Endosialin expression in side populations in human sarcoma cell lines. Oncol Lett 3: 325-329, 2012.

19. Kondo T, Setoguchi T and Taga T: Persistence of a small subpopulation of cancer stem-like cells in the C6 glioma cell line. PNAS 101: 781-786, 2004.

20. Chiba T, Kita K, Zheng YW, et al: Side population purified from hepatocellular carcinoma cells harbors cancer stem cell-like properties. Hepatology 44: 240-251, 2006.

21. Frese KK and Tuveson DA: Maximizing mouse cancer models. Nat Rev Cancer 7: 645-658, 2007.

22. Gibbs CP, Kukekov VG, Reith JD, Tchigrinova O, Suslov ON, Scott EW, GhivizzanisC, Ignatova TN and Steindler DA: Stem-like cells in bone sarcomas: Implications for tumorigenesis. Neoplasia 7: 967-976, 2005.

23. Tirino V, Desiderio V, d'Aquino R, De Francesco F, Pirozzi G, Graziano A, Galderisi U, Cavaliere C, De Rosa A, Papaccio G, et al: Detection and characterization of CD133+cancer stem cells in human solid tumours. PLoS One 3: e3469, 2008 